\title{
Histogram peritumoral enhanced features on MRI arterial phase with extracellular contrast agent can improve prediction of microvascular invasion of hepatocellular carcinoma
}

\author{
Xinxin Wang ${ }^{1 \#}$, Yunfeng Sun ${ }^{1 \#}$, Xueyan Zhou ${ }^{2}$, Zhiwei Shen ${ }^{3}$, Hongxia Zhang ${ }^{1}$, Jiqing Xing ${ }^{4}$, Yang Zhou $^{1}$ \\ ${ }^{1}$ Department of Radiology, Harbin Medical University Cancer Hospital, Harbin, China; ${ }^{2}$ School of Technology, Harbin University, Harbin, China; \\ ${ }^{3}$ Philips Healthcare China, Beijing, China; ${ }^{4}$ Department Physical Education, Harbin Engineering University, Harbin, China \\ Contributions: (I) Conception and design: X Wang, Y Sun, Y Zhou; (II) Administrative support: X Wang, HX Zhang, Y Zhou; (III) Provision of study \\ materials or patients: X Wang, Y Sun, Y Zhou; (IV) Collection and assembly of data: X Wang, Y Sun, J Xing; (V) Data analysis and interpretation: Y \\ Sun, X Zhou, Z Shen; (VI) Manuscript writing: All authors; (VII) Final approval of manuscript: All authors. \\ "These authors contributed equally to this work and should be considered as co-first authors.
}

Correspondence to: Yang Zhou, MD. Department of Radiology, Harbin Medical University Cancer Hospital, 150 Haping Road, Harbin 150010 , China. Email: zhouyang@hrbmu.edu.cn.

Background: Preoperative microvascular invasion (MVI) prediction plays an important role in therapeutic decision-making of hepatocellular carcinoma (HCC). This study aimed to investigate the value of histogram based on the arterial phase (AP) of magnetic resonance imaging (MRI) with extracellular contrast agent compared with radiological features for predicting MVI of solitary HCC.

Methods: In total, 113 patients with pathologically proven solitary HCC were retrospectively enrolled who received surgical resection and underwent preoperative abdominal MRI. The patients were divided into the $\leq 3 \mathrm{~cm}$ [small HCC (sHCC)] cohort and the $>3 \mathrm{~cm}$ cohort. Based on pathological analysis of surgical specimens, the patients were classified into MVI negative (MVI-) and MVI positive (MVI+) groups. Peritumoral and intratumoral histogram features [mean, median, standard deviation (Std), coefficient of variation $(\mathrm{CV})$, skewness, kurtosis] were acquired on AP subtraction images and radiological features [size, capsule, corona enhancement, corona enhancement thickness (CET), CET group]. Receiver operating characteristic (ROC) curve was constructed to assess predictive capability. Subgroup analysis of patients with a visible corona enhancement based on the CET cut-off value was performed.

Results: None of the features extracted from the intratumor area were significantly different between the MVI+ and MVI- groups in both cohorts. Histogram defined peritumoral (peri-) mean, median, kurtosis, and radiological features including CET and CET group were associated with MVI in sHCCs. Peri-mean, median, Std and radiological features including incomplete capsule, CET, and CET group were associated with MVI in HCC $>3 \mathrm{~cm}$. In multivariate logistic regression analysis, the CET group and peri-mean were independent predictors for $\mathrm{HCC}>3 \mathrm{~cm}$ with an area under the curve (AUC) of 0.741 . Peri-mean was an independent predictor for sHCC (AUC $=0.798$ ). Subgroup analysis of the corona enhancement using $8 \mathrm{~mm}$ as a cut-off value showed $100 \%$ sensitivity and negative predictive value (NPV).

Conclusions: Peritumoral AP enhanced degree on MRI showed an encouraging predictive performance for preoperative prediction of MVI, especially in sHCCs. CET $\leq 8 \mathrm{~mm}$ could be used as a negative predictive marker for MVI.

Keywords: Microvascular invasion (MVI); hepatocellular carcinoma (HCC); contrast enhancement; quantitative analysis; magnetic resonance imaging (MRI) 
Submitted May 08, 2021. Accepted for publication Sep 03, 2021.

doi: 10.21037/qims-21-499

View this article at: https://dx.doi.org/10.21037/qims-21-499

\section{Introduction}

Hepatocellular carcinoma (HCC) is the fifth most common type of cancer in China and the second leading cause of cancer-related death worldwide $(1,2)$. Liver resection and transplantation are effective treatments for HCC in patients with preserved liver function (3-5). However, the high recurrence rate of $50-70 \%$ has a significant impact on the long-term survival outcomes after surgery for HCC (6-8). Microvascular invasion (MVI) is one of the crucial potential risk factors for early recurrence of HCC, even for solitary small HCC (sHCC) (9). Therefore, the definitive diagnosis of MVI in HCC preoperatively can guide treatment choice and avoid wasting medical resources.

Contrary to macrovascular invasion that can be detected by diagnostic imaging, MVI is a histologic finding that can only be diagnosed with histopathologic evaluation postoperatively, and preoperative prediction of MVI is still challenging (10). Many studies have reported that MVI of HCC can be predicted by radiological features, such as peritumoral enhancement, corona enhancement, or incomplete capsule, using contrast-enhanced methods such as computed tomography (CT) or dynamic contrastenhanced magnetic resonance imaging (MRI) (11-13). However, these predictors of MVI are far from perfect due to limited information or controversial predictive value. Therefore, more detailed investigations of quantitative information are needed for predicting MVI in HCC.

Histogram analysis is a useful and straightforward quantitative method that can be used to calculate the differentiation of pixel gray distribution and is helpful for radiologists to make a more objective diagnosis $(14,15)$. Studies on HCC have shown that MR histogram analysis has progressed on diagnosis, biologic behavior, and therapy response prediction (16-18). Huang et al. suggested that histogram analysis of MRI may be helpful in detecting MVI of HCC (19). In our previous study, the prediction of MVI was greatly improved through quantitative analysis of morphology on hepatobiliary phase images, but the results through histogram analysis on tumor status to predict MVI were unsatisfactory. Some reports have shown that preoperative MVI predictors were mainly found in the arterial phase (AP), but details on tumor status in the AP of MRI have not been elaborated $(19,20)$. In this study, we aimed to improve the diagnostic value of MVI using histogram features of HCC on the AP images of dynamic contrast-enhanced MRI using extracellular contrast agent.

We present the following article in accordance with the STARD reporting checklist (available at https://dx.doi. org/10.21037/qims-21-499).

\section{Methods}

\section{Patients}

This single-center retrospective study was approved by the Institutional Review Board at our institution and the informed consent requirement was waived. The study was conducted in accordance with the Declaration of Helsinki (as revised in 2013). From our institution's medical database, we identified 170 patients with HCC undergoing surgery and preoperative contrast-enhanced MRI between January 2018 and December 2019. In total, 113 consecutive patients were selected based on the following inclusion criteria: (I) histologically confirmed single HCC without evidence of gross vascular invasion on MRI; (II) no treatment before surgery; (III) gadoteric-acid-enhanced MRI within 14 days before surgery; (IV) HCC with full histological description available in the pathological report; and $(\mathrm{V})$ good image quality (Figure 1).

\section{Clinical and histopathological evaluation}

The clinical data (age, sex and serum markers) were collected from medical records. Pathological characteristics including tumor size, Edmondson-Steiner grade, MVI status and fibrosis stage of background liver from surgical resection specimens were evaluated by two dedicated pathologists ( $>12$ years of experience in abdominal pathology). MVI was defined as the presence of tumor cells in the vascular spaces, indicated by endothelial cell linings, of the surrounding hepatic tissue, microscopically assessed after hematoxylin and eosin (H\&E) staining (Figure 2A-2D).

\section{$M R I$}

All patients underwent contrast-enhanced MRI using a 3.0-Tesla MR system (Ingenia, Philips Healthcare, 


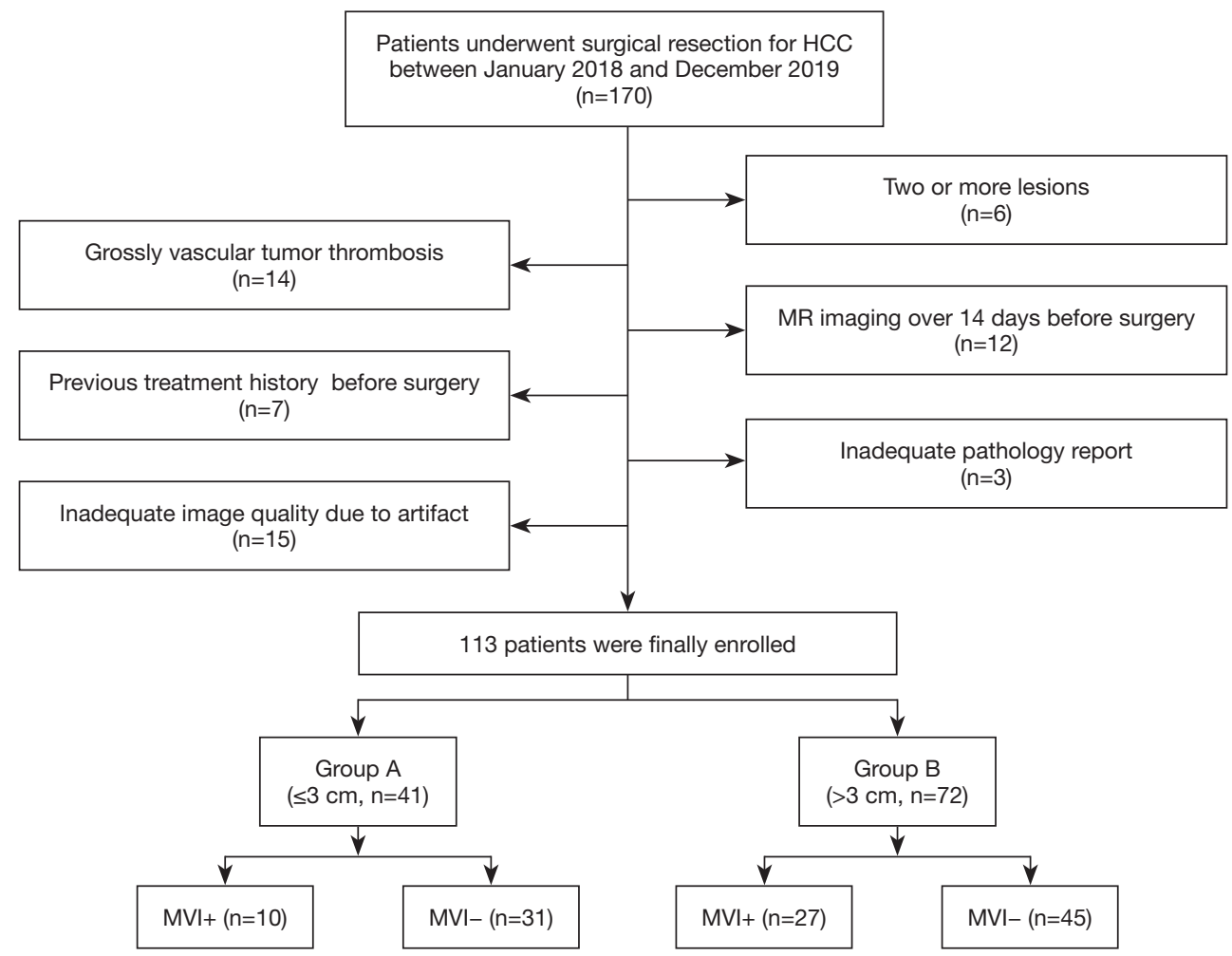

Figure 1 Patient selection for this study. HCC, hepatocellular carcinoma; MVI, microvascular invasion.
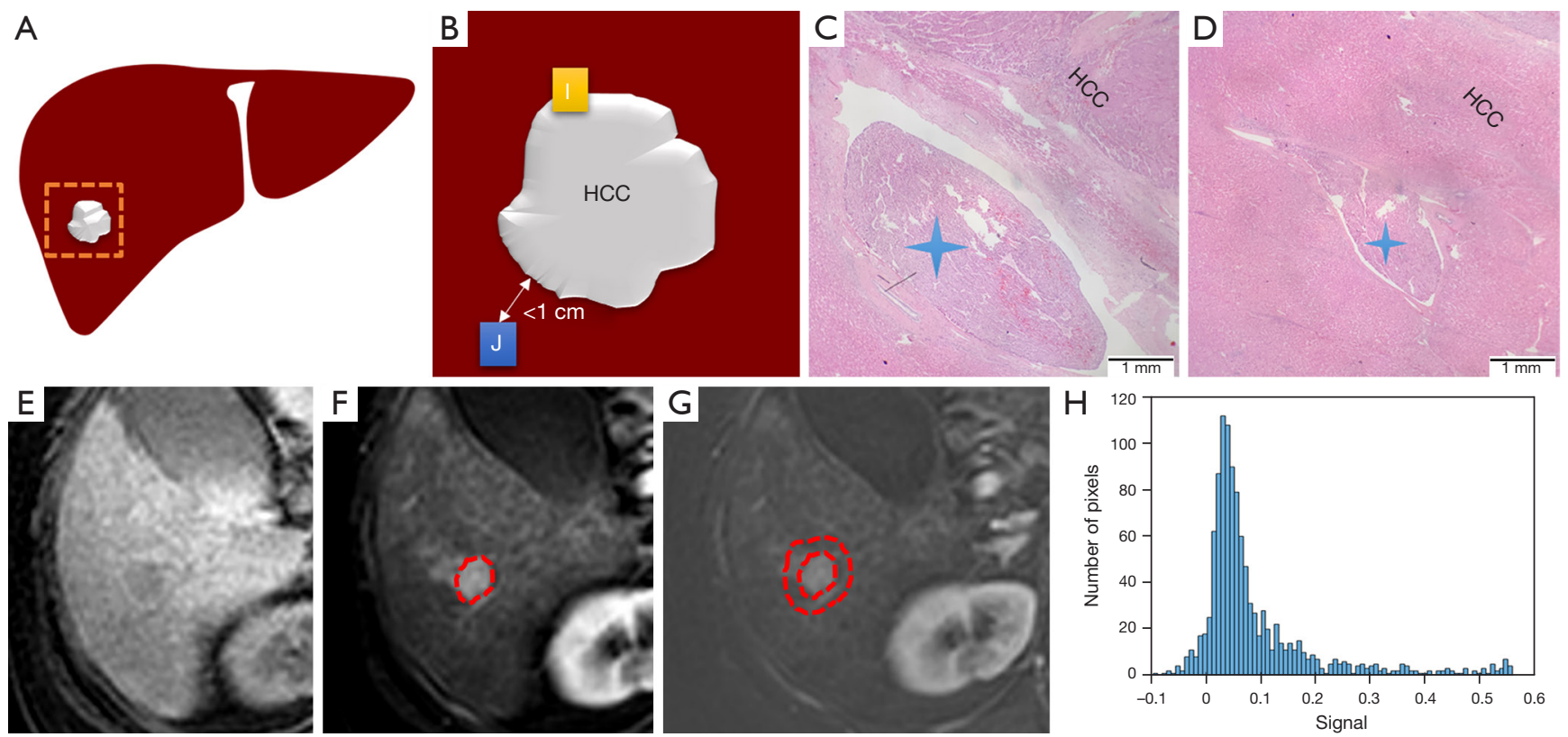

Figure 2 Tumor segmentation for histogram analysis. (A-D) Diagrams of tumor sampling for histology. MVI (asterisk), as identified in H\&Estained sections near the margin of the tumor $[\mathrm{I}]$ and in the peritumoral region $(\leq 1 \mathrm{~cm})[\mathrm{J}]$. The arterial phase image $(\mathrm{F})$ was subtracted from the pre-enhancement image (E) to obtain the subtraction image $(\mathrm{G})$. The red region in the subtraction image $(\mathrm{G})$ within $10 \mathrm{~mm}$ of the tumor contour was automatically created to obtain the histogram (H). HCC, hepatocellular carcinoma; MVI, microvascular invasion. 
Netherlands) with a 32-channel phased-array coil. Firstly, an axial T2-weighted single-shot turbo spin-echo sequence with a fat-suppressed respiratory trigger was performed for routine liver MRI with the following parameters: repetition time (TR)/echo time (TE) $=1,000 / 70 \mathrm{~ms}$, slice thickness/ gap $=7 / 1 \mathrm{~mm}$, field of view $(\mathrm{FOV})=400 \mathrm{~mm} \times 350 \mathrm{~mm}$, and matrix size $=208 \times 207$. Secondly, an axial dual-echo T1-weighted fast field-echo with breath-hold was also performed with TR/TE1/TE2 $=8.7 / 1.15 / 2.3 \mathrm{~ms}$, slice thickness/gap $=7 / 1 \mathrm{~mm}, \mathrm{FOV}=400 \mathrm{~mm} \times 350 \mathrm{~mm}$, and matrix size $=252 \times 211$. Finally, a fat-suppressed dynamic 3D T1-weighted sequence $(\mathrm{TR} / \mathrm{TE}=3.7 / 1.32 \mathrm{~ms}$, slice thickness/gap $=4 /-2 \mathrm{~mm}, \mathrm{FOV}=400 \mathrm{~mm} \times 350 \mathrm{~mm}$, matrix size $=252 \times 207$ ) with volumetric interpolation and breath-hold was performed before and after bolus injection of $0.1 \mathrm{mmol} / \mathrm{kg}$ gadoteric acid (Hengrui Pharmaceutical Co., Ltd., Jiangsu, China) with a flow rate of $2 \mathrm{~mL} / \mathrm{s}$. The dynamic enhanced sequence was performed among several phases including the pre-enhanced phase, AP (25 s), portal venous phase $(55 \mathrm{~s})$, equilibrium phase $(90 \mathrm{~s})$, and delayed phase (180 s).

\section{Evaluation of radiological features on contrast-enhanced MRI}

The conventional T1-weighted dynamic contrast-enhanced images were assessed by two abdominal radiologists (R1 with 3 years and R2 both with 5 years experience). Both radiologists were aware that the lesions were HCC but were blinded to the MVI status and laboratory information. The following MRI features were evaluated by these two radiologists, and identified as potential imaging markers of MVI in previous studies $(11,21)$ : (I) tumor diameter, defined as the maximum diameter on pre-enhanced-phase T1-weighted imaging, classified into two size cohorts $(\leq 3 \mathrm{~cm}$ and $>3 \mathrm{~cm}$ ); (II) corona enhancement, defined as a detectable enhanced portion of a crescent or irregular shape outside the tumor margin with broad contact to the tumor border on the AP, becoming isointense in the portal vein or delayed phase (22); (III) corona enhancement thickness (CET), if the corona enhancement was visible, we measured the maximum perpendicular distance from the tumor boundary to the edge of the corona enhancement; (IV) CET group, we grouped all cases into CET < cutoff value (including non-CET), and > cutoff value; and (V) radiological capsule appearance, categorized as complete, incomplete or absent. If the opinions of the radiologists differed, any disagreement was resolved by consensus.

\section{Quantitative analysis on contrast-enbanced MRI}

For each patient, the tumor outline was traced by a third radiologist with 3 -year of experience (R3) and another radiologist with 5 -year of experience (R4) examined the results. Neither of these radiologists were involved in the evaluation of radiological features. All the quantitative parameters were extracted with an in-house software package based on MATLAB (MathWorks, Natick, MA, USA). Regions of interest (ROIs) were delineated manually on arterial-enhanced-phase images on the largest tumor cross-sectional area by referring to pre-enhanced-phase images. The intratumoral region was defined as the area within the radiologist-labeled tumor boundaries. The peritumoral ROI was dilated at a radius of $1 \mathrm{~cm}$ automatically (it was analyzed as pixel counts multiplied by pixel size) by a topology algorithm from the tumor margin. When the tumor margin was indistinct on arterialphase images, the ROI was drawn after referencing the tumor margin on pre-enhanced or delay-phase images. To observe the real enhancement degree, the final histogram features were extracted from arterial-phase subtraction images (arterial-phase images minus pre-enhanced-phase images), which were mapped from previous intratumoral and peritumoral ROIs. The peritumoral ROI signal intensity histogram parameters were obtained automatically, along with the calculated parameters such as peritumoral mean(peri-mean), peritumoral median(peri-median), peritumoral mode (peri-mode), peritumoral skewness(periskewness), peritumoral kurtosis(peri-kurtosis), peritumoral standard deviation (peri-Std) and peritumoral coefficient of variation (peri-CV). The intratumoral ROI signal intensity histogram parameters were measured and named in the same way (Figure 2E-2H).

\section{Statistical analysis}

According to the maximum tumor diameter, we divided all cases into $\mathrm{sHCC}(\leq 3 \mathrm{~cm})$, and $>3 \mathrm{~cm}$ HCC for analysis. All statistical analyses were performed using SPSS version 22.0 for Windows (SPSS Inc., IL, USA). Statistical significance was set at $\mathrm{P}<0.05$. For data with normal distribution, the independent $t$-test was used to compare continuous variables. The Mann-Whitney $\mathrm{U}$ test was used for continuous data with abnormal distribution. The $\chi^{2}$ test or Fisher's test was used for the categorical variables. Univariate and multivariate analyses were performed by logistic analysis. The receiver operating characteristic 
Table 1 Baseline clinical and histopathological characteristics of the HCC patients

\begin{tabular}{|c|c|c|c|}
\hline Characteristics & $M V I+(n=37)$ & MVI- $(n=76)$ & $P$ \\
\hline \multicolumn{4}{|l|}{ Sex } \\
\hline Male & 31 & 65 & 0.808 \\
\hline Female & 6 & 11 & \\
\hline Mean age (year) & $54.95 \pm 10.01$ & $54.61 \pm 9.23$ & 0.058 \\
\hline \multicolumn{4}{|l|}{ Hepatitis, n (\%) } \\
\hline None & $6(16.2)$ & $7(9.2)$ & 0.291 \\
\hline HBV & $29(78.4)$ & $59(77.6)$ & \\
\hline HCV & $2(5.4)$ & $10(13.2)$ & \\
\hline \multicolumn{4}{|l|}{ Cirrhosis, n (\%) } \\
\hline None & $6(16.2)$ & $19(25.0)$ & 0.291 \\
\hline Yes & $31(83.8)$ & $57(75.0)$ & \\
\hline $\operatorname{AFP}(\mathrm{ng} / \mathrm{mL})$ & $\begin{array}{c}31.50 \\
(5.24-1,210.0)\end{array}$ & $\begin{array}{c}13.24 \\
(4.21-315.95)\end{array}$ & 0.237 \\
\hline AST (U/L) & $50.24 \pm 30.33$ & $45.68 \pm 40.48$ & 0.479 \\
\hline ALT (U/L) & $85.55 \pm 65.46$ & $78.80 \pm 56.45$ & 0.999 \\
\hline GGT (U/L) & $81.30 \pm 68.33$ & $76.47 \pm 54.35$ & 0.406 \\
\hline \multicolumn{4}{|c|}{ Child-Pugh class, n (\%) } \\
\hline A & $33(89.2)$ & $68(89.5)$ & 0.963 \\
\hline B & $4(10.8)$ & $8(10.5)$ & \\
\hline \multicolumn{4}{|c|}{ BCLC stage, n (\%) } \\
\hline $\mathrm{AO}$ & $0(0)$ & $8(10.5)$ & 0.059 \\
\hline $\mathrm{A} 1$ & $15(40.5)$ & $41(53.9)$ & \\
\hline $\mathrm{A} 2$ & $3(8.1)$ & $3(3.9)$ & \\
\hline A3 & $2(5.4)$ & $5(6.6)$ & \\
\hline $\mathrm{B}$ & $17(45.9)$ & $19(25)$ & \\
\hline \multicolumn{4}{|c|}{ Differentiation grade, $\mathrm{n}(\%)$} \\
\hline Low & $5(13.5)$ & $7(9.2)$ & 0.256 \\
\hline Moderate & $3(8.1)$ & $15(19.7)$ & \\
\hline High & $29(78.4)$ & $54(71.1)$ & \\
\hline Tumor size $(\mathrm{cm})$ & $5.00(3.00-7.50)$ & $3.50(2.50-5.00)$ & $0.025^{\star}$ \\
\hline
\end{tabular}

Continuous variables are presented as means \pm standard deviations. Categorical variables are presented as $n(\%)$. Significant results are shown by asterisk ( $\left.{ }^{*}, \mathrm{P}<0.05\right)$. HCC, hepatocellular carcinoma; MVI, microvascular invasion; HBV, hepatitis B virus; HCV, hepatitis C virus; AFP, alpha-fetoprotein; $A S T$, aspartate aminotransferase; ALT, alanine aminotransferase; GGT, gamma-glutamyltransferase; BCLC, Barcelona Clinic Liver Cancer.
(ROC) curve was drawn to determine the cut-off value of the parameters using the maximum Youden index. For each ROC curve, the area under the curve (AUC) was calculated. The DeLong test was performed to compare AUCs. The sensitivity, specificity, positive predictive value (PPV) and negative predictive value (NPV) were calculated. The interobserver variability for radiological features was assessed using the Kappa analysis $(\kappa=0.00-0.20$, poor agreement; $\kappa=0.21-0.40$, fair agreement; $\kappa=0.41-0.60$, moderate agreement; $\kappa=0.61-0.80$, good agreement; $\kappa=0.81-1.0$, excellent agreement).

\section{Results}

\section{Clinical data analysis}

Clinical and pathological features are shown in Table 1. Thirty-seven (32.7\%) patients had pathologically confirmed MVI+ HCC. In all tumors, tumor size in MVI+ patients was significantly larger than in MVI- patients. There were no significant differences in sex, age, hepatitis, cirrhosis, $\alpha$-fetoprotein, aspartate aminotransferase, alanine aminotransferase, $\gamma$-glutamyltransferase, Child-Pugh class, or differentiation grade between MVI+ and MVI- in all tumors.

\section{Evaluation of radiological features}

The interobserver agreement between the two radiologists was high using radiological features to predict MVI. The level of agreement for capsule and corona enhancement was excellent (kappa: 0.812 and 0.871) and the agreement for thickness of the corona enhancement and irregular rim enhancement was good (kappa: 0.665 and 0.715). The results for further research were consensus decisions. Among the MRI features, visibility of the corona enhancement had no significant effect on the prediction of MVI. However, CET was able to predict MVI in both cohorts: sHCC $(\mathrm{P}=0.001)$ and HCC $>3 \mathrm{~cm}(\mathrm{P}=0.009)$. Furthermore, the optimal cut-off value of $8 \mathrm{~mm}$ for CET was acquired from ROC analysis according to the Youden index, and we divided all cases into two groups [CET $>8 \mathrm{~mm}$; CET $\leq 8 \mathrm{~mm}$ (including absent)] (Figure 3). There were statistically significant differences for the CET group in predicting MVI in sHCC $(\mathrm{P}=0.034)$ and $\mathrm{HCC}>3 \mathrm{~cm}$ $(\mathrm{P}=0.046)$, respectively. There was also the difference in the incomplete capsule between MVI+ and MVI- in the HCC $>3 \mathrm{~cm}$ cohort $(\mathrm{P}=0.022)$. These results are shown in Table 2 . 

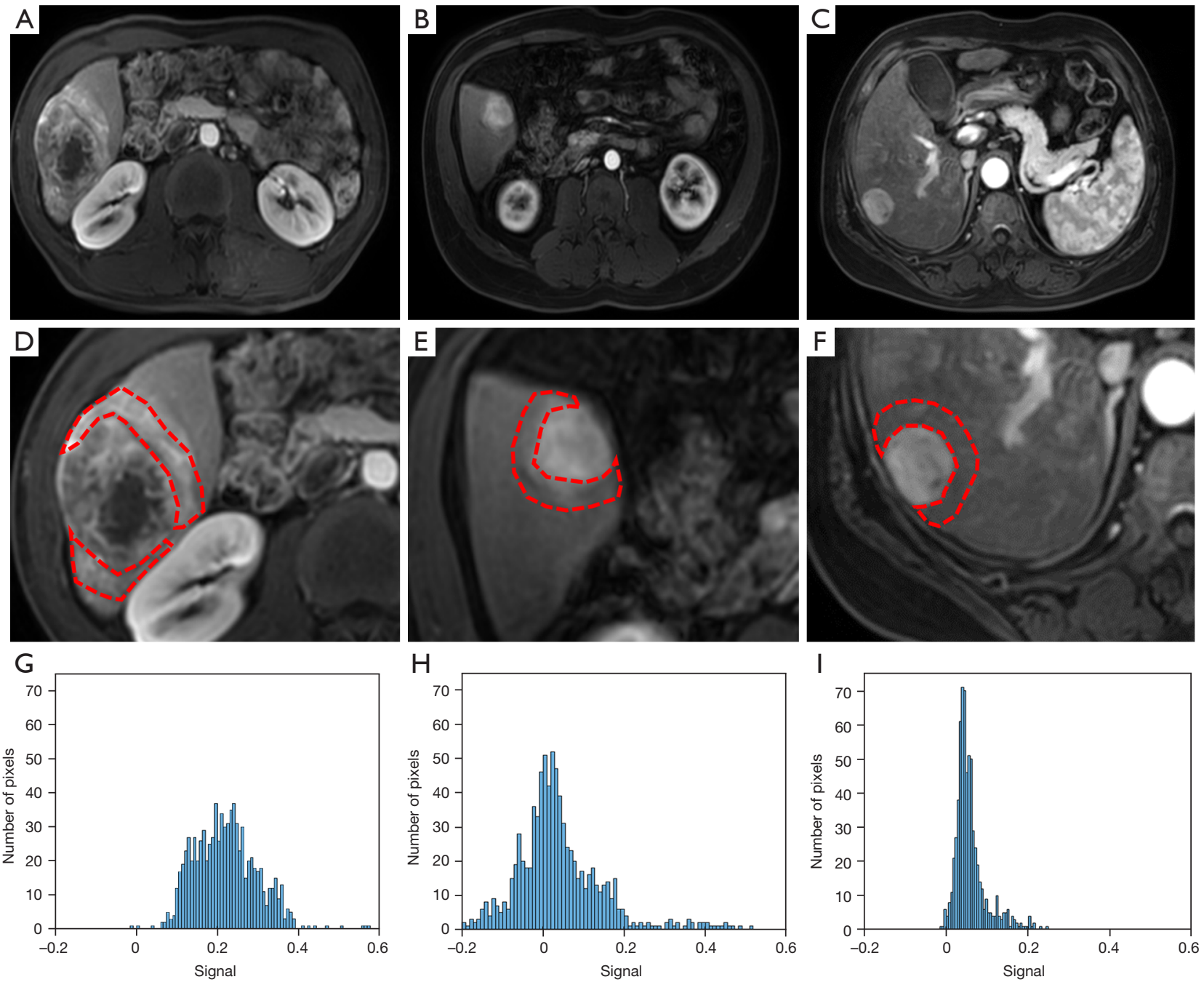

Figure 3 Arterial-phase MR image and differences in distribution of histogram parameters between HCC with and without MVI. (A) Arterial-phase MR image shows HCC with MVI+ and CET $>8 \mathrm{~mm}$. (B) Arterial-phase MR image shows HCC $>3 \mathrm{~cm}, \mathrm{MVI}-$ with CET $<8 \mathrm{~mm}$. (C) Arterial-phase MR image shows sHCC (size $\leq 3 \mathrm{~cm}$ ), MVI+ without corona enhancement. (D) Peritumoral ROI arterial-phase subtraction images for (A). (E) Peritumoral ROI arterial-phase subtraction images for (B). (F) Peritumoral ROI arterial-phase subtraction images for (C). (G) Histogram for (D). (H) Histogram for (E). (I) Histogram for (F). HCC with MVI (G,I) shows a relative frequency at higher signal than that of HCC without MVI (H). The red region in (D-F) indicate peritumoral ROI. HCC, hepatocellular carcinoma; MVI, microvascular invasion; CET, corona enhancement thickness; sHCC, small HCC; ROI, regions of interest.

\section{Histogram evaluation}

In both two cohorts (sHCC and HCC $>3 \mathrm{~cm}$ ), significant differences were noted in peri-mean and peri-median calculated from arterial-phase subtraction images between MVI+ and MVI- in all tumors. There were also significant differences in peri-kurtosis in the sHCC, and peri-Std in the HCC $>3 \mathrm{~cm}$ cohorts. There were no significant differences in any intratumoral parameters. These results are shown in
Table 3 and Figure 4.

\section{Univariate and multivariate logistic regression analyses}

The univariate and multivariate analyses are summarized in Table 4. In the sHCC, peri-mean, peri-median and perikurtosis based on arterial-phase subtraction images, CET and CET group (grouping by CET $=8 \mathrm{~mm}$ ) were included 
Table 2 Differences in radiologic features in HCC patients with and without MVI

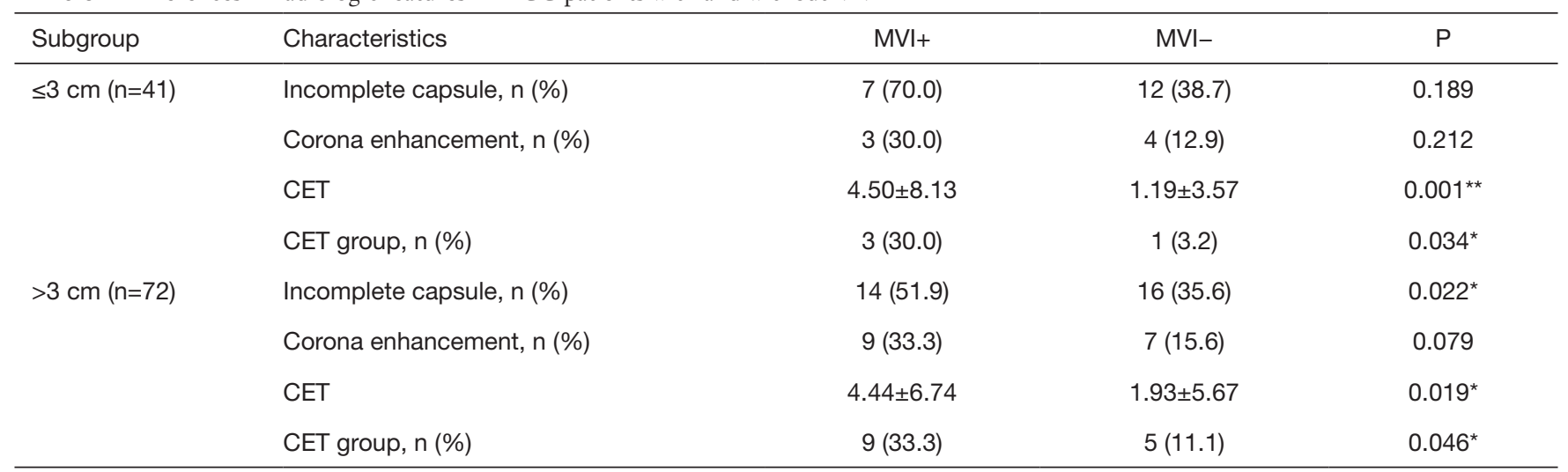

Data shown as $\mathrm{n}(\%)$ or mean \pm standard deviation. Significant results are shown by asterisks $\left({ }^{*}, \mathrm{P}<0.05 ;{ }^{* *}, \mathrm{P}<0.01\right)$. HCC, hepatocellular carcinoma; MVI, microvascular invasion; CET, corona enhancement thickness, defined as the maximum vertical distance from the boundary of the tumor to the edge of the corona enhancement; CET group, the corona enhancement thickness group, was divided into three parts (none, present and less than $8 \mathrm{~mm}$, present and larger than $8 \mathrm{~mm}$ ).

Table 3 Differences in histogram parameters of peritumor and intratumor in HCC patients with and without MVI

\begin{tabular}{|c|c|c|c|c|c|c|}
\hline Parameters & \multicolumn{3}{|c|}{$\mathrm{HCC} \leq 3 \mathrm{~cm}(\mathrm{n}=41)$} & \multicolumn{3}{|c|}{$\mathrm{HCC}>3 \mathrm{~cm}(\mathrm{n}=72)$} \\
\hline Peri_mean & $0.19 \pm 0.16$ & $0.09 \pm 0.05$ & $0.003^{\star *}$ & $0.12 \pm 0.75$ & $0.73 \pm 0.47$ & $0.001^{* *}$ \\
\hline Peri_median & $0.13 \pm 0.07$ & $0.08 \pm 0.05$ & $0.006^{\star *}$ & $0.11 \pm 0.07$ & $0.06 \pm 0.05$ & $0.005^{\star *}$ \\
\hline Peri_Std & $0.07 \pm 0.04$ & $0.05 \pm 0.02$ & 0.065 & $0.06 \pm 0.03$ & $0.05 \pm 0.02$ & $0.006^{* *}$ \\
\hline Peri_skewness & $0.58 \pm 0.90$ & $1.15 \pm 0.69$ & 0.059 & $1.08 \pm 0.84$ & $1.16 \pm 0.74$ & 0.677 \\
\hline Peri_kurtosis & $3.85 \pm 1.83$ & $5.41 \pm 2.12$ & $0.043^{*}$ & $5.85 \pm 3.00$ & $6.28 \pm 3.53$ & 0.596 \\
\hline Intra_mean & $0.21 \pm 0.08$ & $0.21 \pm 0.09$ & 0.911 & $0.16 \pm 0.09$ & $0.16 \pm 0.13$ & 0.945 \\
\hline Intra_median & $0.22 \pm 0.08$ & $0.22 \pm 0.10$ & 0.997 & $0.16 \pm 0.10$ & $0.15 \pm 0.11$ & 0.681 \\
\hline Intra_skewness & $0.44 \pm 0.44$ & $0.28 \pm 0.74$ & 0.522 & $0.20 \pm 0.68$ & $0.22 \pm 0.80$ & 0.918 \\
\hline Intra_kurtosis & $3.01 \pm 0.41$ & $3.43 \pm 1.12$ & 0.255 & $3.52 \pm 1.12$ & $3.97 \pm 1.92$ & 0.271 \\
\hline
\end{tabular}

Data shown as mean \pm standard deviation. Significant results are shown by asterisks $\left({ }^{*}, \mathrm{P}<0.05 ;{ }^{* \star}, \mathrm{P}<0.01\right)$. HCC, hepatocellular carcinoma; MVI, microvascular invasion; Peri, peritumoral; Intra, intratumoral; Std, standard deviation; CV, coefficient of variation.

in the multivariate analysis. Peri-mean $(\mathrm{P}=0.004)$ was the only independent predictive factor for MVI in the sHCC cohort. In the HCC $>3 \mathrm{~cm}$ cohort, mean, median, and Std of the peritumoral region and three radiological features (incomplete capsule, CET and CET group) were included in the multivariate analysis. Finally, peri-mean $(\mathrm{P}=0.003)$ and CET group $(\mathrm{P}=0.048)$ were identified as independent predictive factors of MVI (Figure $3 A, 3 B, 3 G, 3 H$ ).

\section{Prediction of MVI}

The ROC curves based on the selected independent factors in sHCC and HCC $>3 \mathrm{~cm}$ are shown in Figure 5. The parameters including AUC, sensitivity and specificity for each 

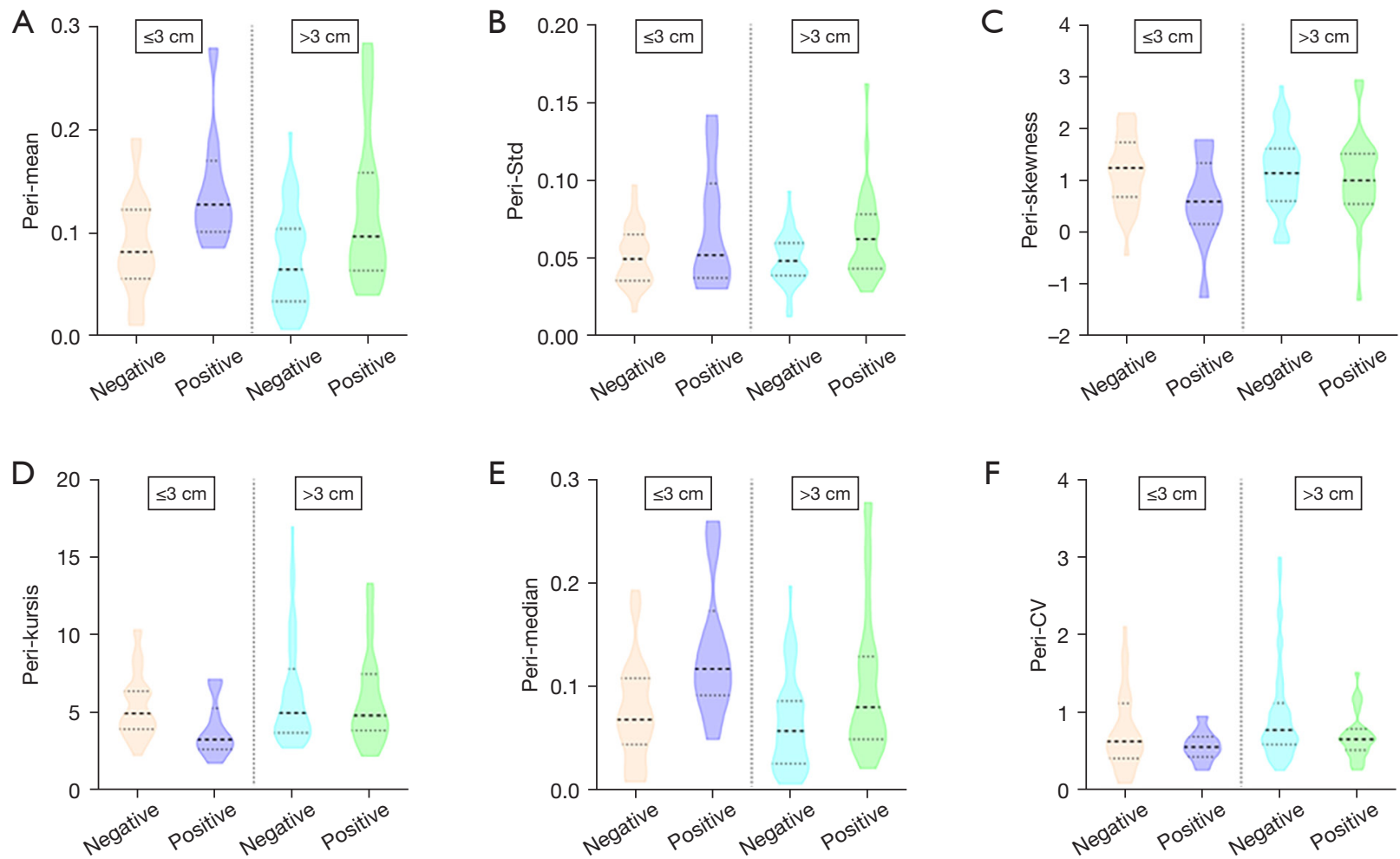

Figure 4 Violin plots of parameters for the MVI negative (MVI-) and MVI positive (MVI+) groups in $\mathrm{sHCC}$ (size $\leq 3 \mathrm{~cm}$ ) and $\mathrm{HCC}>3 \mathrm{~cm}$. (A) Peri-mean, (B) peri-std, (C) peri-skewness, (D) peri-kurtosis, (E), peri-median, (F) peri-CV. The peri-mean and peri-median average values for MVI+ were significantly higher than MVI- in both the sHCC and HCC $>3 \mathrm{~cm}$ groups $(\mathrm{P}<0.01)$. The peri-Std average value for MVI+ was significantly higher than MVI- in the HCC $>3 \mathrm{~cm}(\mathrm{P}=0.006)$ group, but was not significantly different for MVI+ and MVI- in sHCC $(\mathrm{P}=0.065)$. The peri-kurtosis average value for MVI+ was significantly higher than that for MVI- in sHCC ( $\mathrm{P}=0.043)$ but was not significantly different for MVI+ and MVI- in the HCC $>3 \mathrm{~cm}$ group $(\mathrm{P}=0.596)$. The peri-CV and peri-skewness average value were not significantly different for MVI+ and MVI- in all HCCs. MVI, microvascular invasion; HCC, hepatocellular carcinoma; sHCC, small HCC; peritumoral mean, peri-mean; peritumoral median, peri-median; peritumoral skewness, peri-skewness, peritumoral kurtosis, peri-kurtosis; peritumoral standard deviation, peri-Std; peri-CV, peritumoral coefficient of variation.

predictor are listed in Table 5. In the sHCC cohort, peri-mean alone had an AUC of $0.792(\mathrm{P}=0.0001)$ with a sensitivity and specificity of $100.00 \%$ and $54.84 \%$, respectively (Figure $5 A$ ). When the peri-mean and CET group were combined, the AUC improved to 0.741 in the HCC $>3 \mathrm{~cm}$ group, which was better than the CET group alone $(\mathrm{AUC}=0.611 ; \mathrm{P}=0.01$, DeLong test) but not significantly higher than the perimean with the AUC of 0.705 ( $\mathrm{P}=0.360$, DeLong test) (Figure $5 B)$. When the univariate parameters from both sHCC and $\mathrm{HCC}>3 \mathrm{~cm}$ were pooled for the multiple logistic regression analysis (Figure 5C), peri-mean (OR, 6.198; 95\% CI, 1.48215.382) and CET group (OR, 5.191; 95\% CI, 1.324-7.771) were significant factors for MVI in all tumors with an AUC of 0.716 and 0.623 , respectively (Figure $5 D$ ). When the above two features were combined, the AUC was 0.756 with a sensitivity and specificity of $59.46 \%$ and $77.63 \%$, respectively.

\section{CET subgroup analysis}

According to the CET, we performed a subgroup analysis with the corona enhancement visible cohort. CET $>8$ or $\leq 8 \mathrm{~mm}$, showed a significant difference $[\mathrm{P}=0.01, \mathrm{OR}, 3.0$ (1.56-5.77)] between MVI+ and MVI- with an AUC 0.727, sensitivity $100 \%$, specificity $45.45 \%$, PPV $66.7 \%$, and NPV $100 \%$ (Table 6, Figure 3A,3C,3D,3F).

\section{Discussion}

In this retrospective study, we established a quantitative analytical method to predict MVI in patients with HCC 
Table 4 Univariate and multivariate analyses of quantitative analysis and radiologist analysis

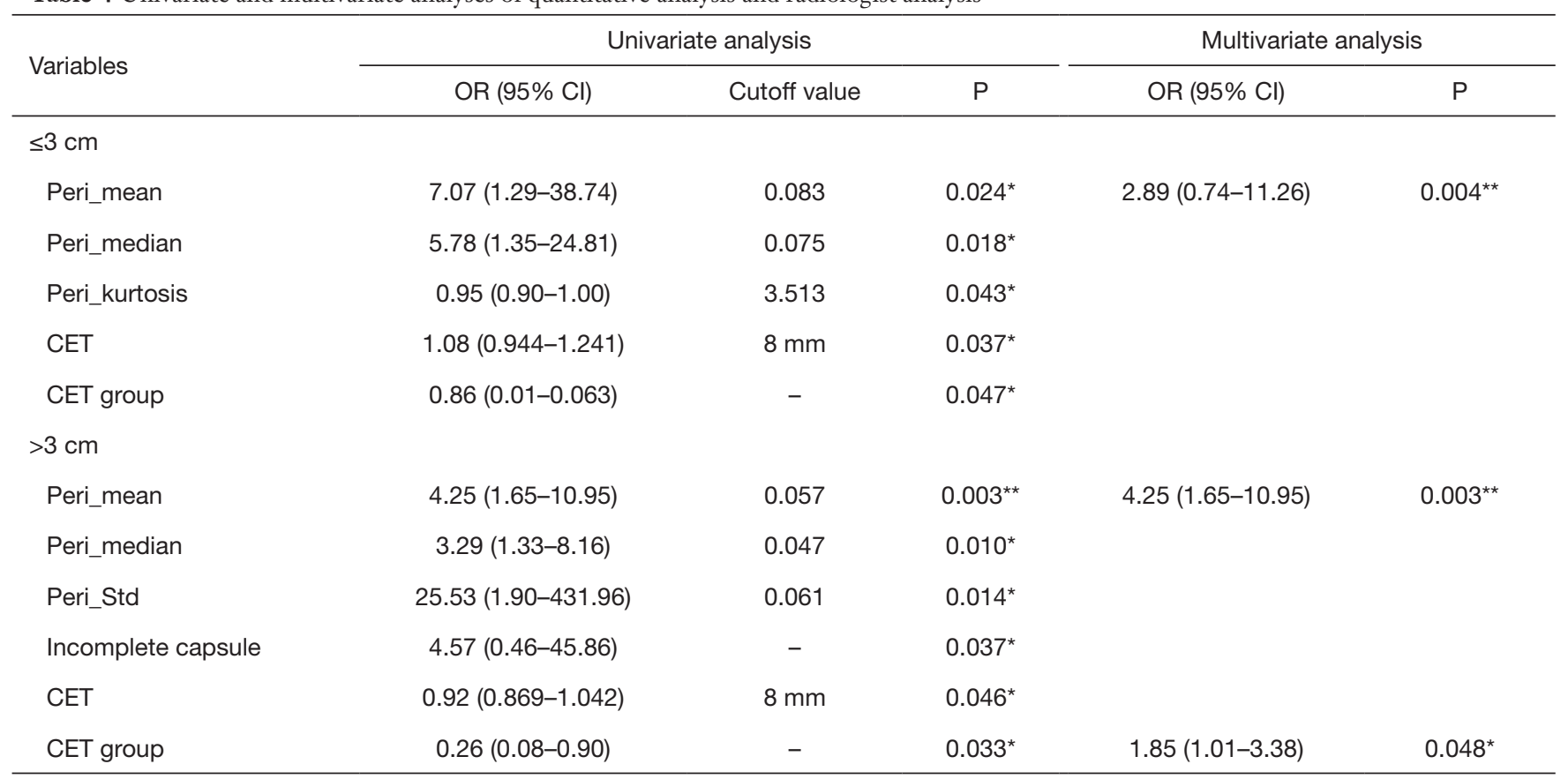

Significant results are shown by asterisks $\left({ }^{*}, \mathrm{P}<0.05\right.$, $\left.{ }^{\star *}, \mathrm{P}<0.01\right)$. Variables with $\mathrm{P}<0.05$ in univariate logistic regression analysis were included in the multivariate logistic regression analysis. OR, odds ratio; Peri, peritumoral; Std, standard deviation; CET, corona enhancement thickness, defined as the maximum vertical distance from the boundary of the tumor to the edge of the corona enhancement; CET group, the corona enhancement thickness group, was divided into two parts (none, present and less than $8 \mathrm{~mm}$; present and larger than $8 \mathrm{~mm}$ ).

based on arterial-phase MRI with extracellular contrast agent. Our results indicated that the mean enhanced degree of the peritumoral region showed better predictive performance, especially for sHCC. Compared with the perimean, the combination of parameters including CET group and peri-mean did not significantly improve the predictive power in $\mathrm{HCC}>3 \mathrm{~cm}$ and all tumor sizes.

Histogram parameters from intratumoral and peritumoral regions of HCC were analyzed to examine their possible relationship with MVI. There was no significant difference in histogram features between the MVI+ and MVI- groups in intratumoral regions, which was different to the results of Huang et al. (19). We speculated that the reason of the difference was using arterial-phase subtraction images. Therefore, removing interference from T1-weighted inherent signaling (such as hemorrhage, cystic degeneration, necrosis), and only analyzed the tumor enhancement characteristics. Our results indicated that the intratumoral enhancement quantitative features were not significantly correlated with MVI in both groups. In addition, the autorecognition algorithm was used to perform the ROIs extended to a radius of $1 \mathrm{~cm}$ from the tumor margin with regard to the pathological sampling criteria. The histogram characteristics of this region showed significant correlations with MVI.

Occasionally, the subtle signal changes are not easily visible surrounding a tumor for radiologists, histogram features can provide more imaging details and assist doctors in diagnosis as a more sensitive quantitative approach $(14,15,19,23)$. In this study, peri-mean was an independent predictor of MVI in both cohorts ( $\mathrm{sHCC}$ and $\mathrm{HCC}>3 \mathrm{~cm}$ ). These results suggested that the mean degree of the peritumoral enhancement signal was associated with MVI: With the increase in peri-mean value, the possibility of MVI was greater. It may be related to peritumoral hemodynamic changes caused by positive MVI, and the enhancement degree of peritumoral is enhanced, reflecting the increase of peri-mean. Particularly in sHCC, only the peri-mean feature is taken as an independent predictive factor, and the diagnostic efficiency achieved a satisfactory result (AUC: 0.798). In addition, the sensitivity was up to $100 \%$, which may have significant clinical implications. Therefore, for sHCC patients, we suggest that a more cautious treatment strategy should be chosen for those 

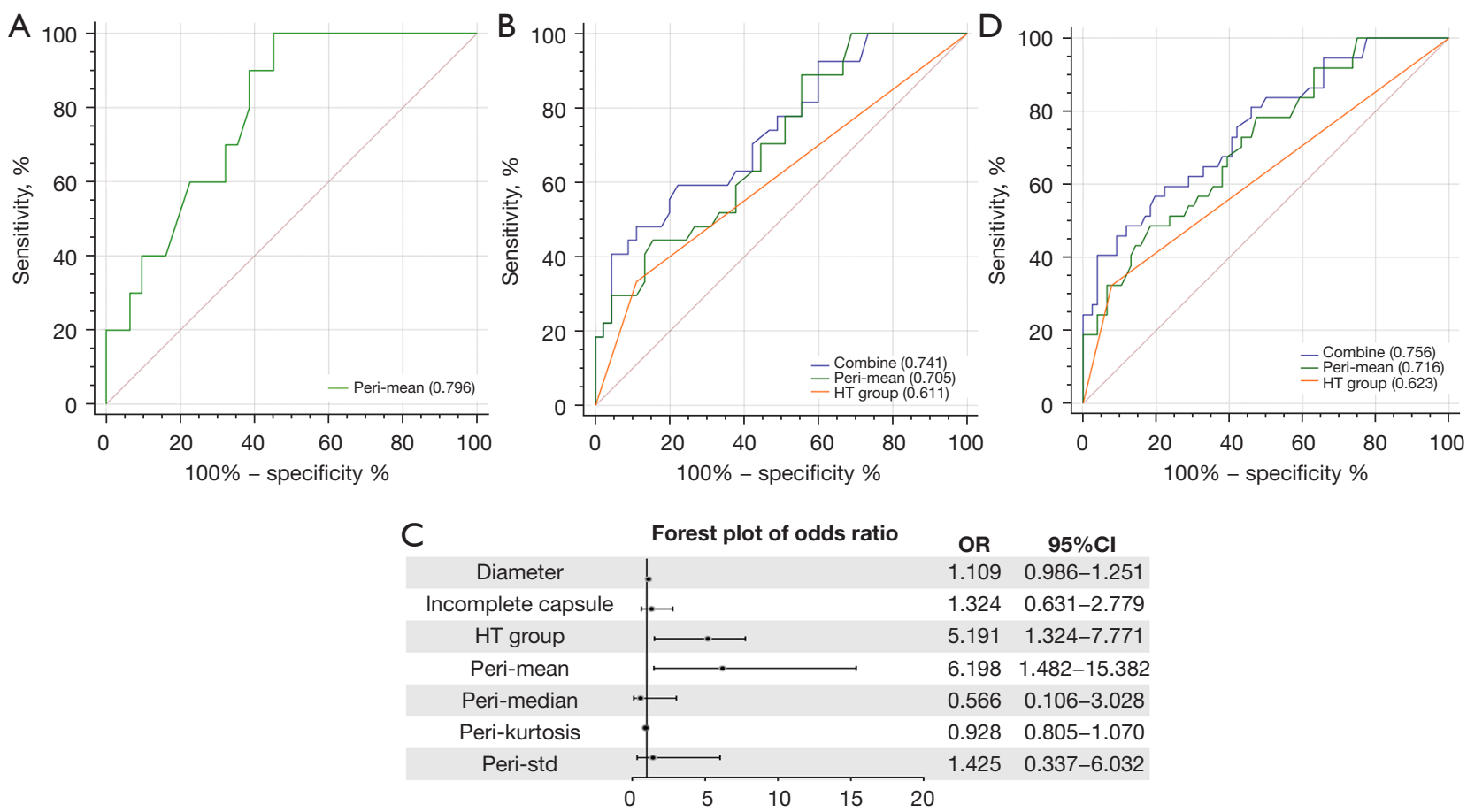

Figure 5 Comparison of ROC curves in predicting MVI for sHCC, HCC $>3 \mathrm{~cm}$ and OR and $95 \%$ CI on the basis of multivariate logistic regression for each parameter. (A). In sHCC, the AUC of the peri-mean was 0.792. (B) In HCC $>3 \mathrm{~cm}$, the AUC of the combined model (AUC $=0.741$ ) was higher than that of the peri-mean (AUC =0.705) and CET group (AUC =0.611), respectively. (C) OR and 95\% CI on the basis of multivariate logistic regression for both $\mathrm{sHCC}$ and $\mathrm{HCC}>3 \mathrm{~cm}$. (D) In all tumors, the combined model demonstrated higher predictive ability with an AUC 0.756 than the peri-mean (AUC =0.716) and CET group (AUC =0.623), respectively. ROC, receiver operating characteristic; MVI, microvascular invasion; HCC, hepatocellular carcinoma; sHCC, small HCC; OR, odds ratio; AUC, area under the curve; CET, corona enhancement thickness; CI, confidence interval.

with a peri-mean value higher than 0.083 (cutoff value), to avoid postoperative recurrence caused by non-anatomical hepatectomy. In addition, radiofrequency ablation was also not recommended for these sHCC patients.

The corona enhancement is a vital evaluation parameter on the AP, and was highly suggestive for predicting MVI in previous studies $(11,24)$. However, this approach remains controversial. Reginelli et al. demonstrated that peritumoral enhancement was a non-significant factor for MVI in the hepatic AP of CT scans, similar to our results (25). We hypothesized that this may be due to the lower incidence of the corona enhancement on AP images in HCC patients. In the current study, incidence of the corona enhancement was merely $20.35 \%(23 / 113)$ in all cases, $17.07 \%$ in sHCC (7/41), which could have influenced the diagnostic efficiency, especially the sensitivity. We further measured the thickness of the corona enhancement as a new variable, which has not been mentioned in previous studies, and found it was significantly related to MVI in univariate analysis, but not in multivariate analysis. When we regarded the cut-off value $(8 \mathrm{~mm})$ as the group value to divide cases into two groups [ $\leq 8 \mathrm{~mm}$ (including absent) and $>8 \mathrm{~mm}$ ], the CET group was another independent predictor in the HCC $>3 \mathrm{~cm}$ group besides the peri-mean. In addition, we selected all corona-visible cases to perform a subgroup analysis. An NPV of $100 \%$ in HCCs with a CET $\leq 8 \mathrm{~mm}$ was obtained. Therefore, we suggest the following two points regarding the corona enhancement: Firstly, the diagnosis of MVI by the corona enhancement alone was not sufficient, CET should also be considered; secondly, CET $<8 \mathrm{~mm}$ may be an essential negative biological marker of MVI. Therefore, curative resection should be carefully considered to avoid unnecessary liver transplantation and reduce wastage of medical resources for cases with a relatively smaller corona enhancement after excluding other risk factors.

It is known that tumor size may guide different clinical 
Table 5 Comparison of diagnostic performance of independent predictors for MVI of different sizes

\begin{tabular}{|c|c|c|c|c|c|}
\hline Subgroup & AUC (95\% Cl) & Sensitivity & Specificity & PPV & NPV \\
\hline Peri_mean & $0.798(0.644-0.907)$ & $54.84 \%$ & $100 \%$ & $41.7 \%$ & $100 \%$ \\
\hline \multicolumn{6}{|l|}{$>3 \mathrm{~cm}$} \\
\hline Peri_mean & $0.705(0.586-0.807)$ & $100.00 \%$ & $31.11 \%$ & $46.60 \%$ & $100.00 \%$ \\
\hline Combined & $0.741(0.683-0.880)$ & $88.89 \%$ & $55.56 \%$ & $54.50 \%$ & $89.30 \%$ \\
\hline \multicolumn{6}{|l|}{ All } \\
\hline Peri_mean & $0.716(0.625-0.798)$ & $78.38 \%$ & $52.63 \%$ & $44.60 \%$ & $83.30 \%$ \\
\hline CET group & $0.623(0.406-0.597)$ & $32.43 \%$ & $92.11 \%$ & $66.70 \%$ & $33.70 \%$ \\
\hline
\end{tabular}

$\mathrm{MVI}$, microvascular invasion; AUC, area under receiver operating characteristic curve; Cl, confidence interval; PPV, positive predictive value; NPV, negative predictive value; Peri, peritumoral; CET group, the corona enhancement thickness group, was divided into two parts (none, present and less than $8 \mathrm{~mm}$; present and larger than $8 \mathrm{~mm}$ ).

Table 6 Corona enhancement thickness subgroup analysis

\begin{tabular}{lccc}
\hline Corona enhancement thickness & $\mathrm{MVI}+(\mathrm{n}=12)$ & $\mathrm{MVI}-(\mathrm{n}=11)$ & OR $(95 \% \mathrm{Cl})$ \\
\hline$\leq 8 \mathrm{~mm}$ & $0(0.0 \%)$ & $5(45.55 \%)$ & $3.00(1.56-5.77)$ \\
$>8 \mathrm{~mm}$ & $12(100 \%)$ & $6(54.5 \%)$ & \\
\hline
\end{tabular}

Sensitivity, 100\%; specificity, 45.45\%; PPV, 66.7\%; NPV, 100\%; AUC, 0.727 (0.573-0.882). MVI, microvascular invasion; PPV, positive predictive value; PPV, positive predictive value; AUC, area under receiver operating characteristic curve.

therapeutic management $(26,27)$. However, few studies have discussed MVI in groups according to tumor size $(28,29)$. Our results showed that different features predicted MVI in the $\mathrm{sHCC}$ and HCC $>3 \mathrm{~cm}$ cohorts. Peri-mean was the most valuable MVI predictor for HCCs, especially for sHCCs. However, it should be noted that CET was also a predictor in the HCC $>3 \mathrm{~cm}$ cohort. Although the predictive power of the combination of these two parameters was not statistically significant compared with peri-mean alone $(\mathrm{P}=0.19$, DeLong test), the combination still improved the diagnostic performance (AUC $=0.756$ ) to some extent for $\mathrm{HCC}>3 \mathrm{~cm}$. Therefore, individualized evaluation criteria for different sized tumors should be proposed, which would guide more appropriate treatment options for HCC patients.

There were some limitations associated with this study. Firstly, the sample size was small and restricted to solitary HCC, especially sHCCs. Secondly, only three radiological features based on enhanced images were used to compare with the results from peritumoral region quantitative analysis, without importing other multi-parameters such as
T2-weighted and diffusion-weighted imaging. Finally, the maximal cross-sectional areas of the tumors were analyzed instead of the whole volume of the lesions, even though it was the main observation slice for the radiologist and could provide more intuitive information, especially in small entities. Thus, further research with a large sample in a multicenter setting is necessary to prove the diagnostic power of the metrics identified and further radiomic analyze.

In conclusion, peritumoral histogram features on arterial-phase subtraction MRI can provide more confident prediction efficiency of MVI in HCC. Peritumoral mean enhanced degree on the histogram is a significant parameter for improving prediction of MVI, especially for sHCC. CET should also be considered rather than whether the corona enhancement is visible for MVI diagnosis in patients with $\mathrm{HCC}>3 \mathrm{~cm}$.

\section{Acknowledgments}

Funding: This study received funding by the 2020 SKY 
Imaging Research Fund of the Chinese International Medical Foundation (project No. Z-2014-07-2003-07); Harbin Medical University Cancer Hospital Haiyan Funds (No. JJZD2020-17). Harbin Medical University Cancer Hospital Haiyan Funds (No. JJQN2021-07).

\section{Footnote}

Reporting Checklist: The authors have completed the STARD reporting checklist. Available at https://dx.doi. org/10.21037/qims-21-499

Conflicts of Interest: All authors have completed the ICMJE uniform disclosure form (available at https://dx.doi. org/10.21037/qims-21-499). The authors have no conflicts of interest to declare.

Ethical Statement: The authors are accountable for all aspects of the work in ensuring that questions related to the accuracy or integrity of any part of the work are appropriately investigated and resolved. The study was conducted in accordance with the Declaration of Helsinki (as revised in 2013). This single-center retrospective study was approved by the Institutional Review Board at our institution and the informed consent requirement was waived.

Open Access Statement: This is an Open Access article distributed in accordance with the Creative Commons Attribution-NonCommercial-NoDerivs 4.0 International License (CC BY-NC-ND 4.0), which permits the noncommercial replication and distribution of the article with the strict proviso that no changes or edits are made and the original work is properly cited (including links to both the formal publication through the relevant DOI and the license). See: https://creativecommons.org/licenses/by-nc-nd/4.0/.

\section{References}

1. Torre LA, Bray F, Siegel RL, Ferlay J, Lortet-Tieulent J, Jemal A. Global cancer statistics, 2012. CA Cancer J Clin 2015;65:87-108

2. Chen W, Zheng R, Baade PD, Zhang S, Zeng H, Bray F, Jemal A, Yu XQ, He J. Cancer statistics in China, 2015. CA Cancer J Clin 2016;66:115-32.

3. Bruix J, Reig M, Sherman M. Evidence-Based Diagnosis, Staging, and Treatment of Patients With Hepatocellular Carcinoma. Gastroenterology 2016;150:835-53.
4. European Association for the Study of the Liver. EASL Clinical Practice Guidelines: Management of hepatocellular carcinoma. J Hepatol 2018;69:182-236.

5. Romagnoli R, Mazzaferro V, Bruix J. Surgical resection for hepatocellular carcinoma: Moving from what can be done to what is worth doing. Hepatology 2015;62:340-2.

6. Fujiwara N, Friedman SL, Goossens N, Hoshida Y. Risk factors and prevention of hepatocellular carcinoma in the era of precision medicine. J Hepatol 2018;68:526-49.

7. Forner A, Reig M, Bruix J. Hepatocellular carcinoma. Lancet 2018;391:1301-14.

8. Burrel M, Reig M, Forner A, Barrufet M, de Lope CR, Tremosini S, Ayuso C, Llovet JM, Real MI, Bruix J. Survival of patients with hepatocellular carcinoma treated by transarterial chemoembolisation (TACE) using Drug Eluting Beads. Implications for clinical practice and trial design. J Hepatol 2012;56:1330-5.

9. Sumie S, Kuromatsu R, Okuda K, Ando E, Takata A, Fukushima N, Watanabe Y, Kojiro M, Sata M. Microvascular invasion in patients with hepatocellular carcinoma and its predictable clinicopathological factors. Ann Surg Oncol 2008;15:1375-82.

10. Zhang X, Li J, Shen F, Lau WY. Significance of presence of microvascular invasion in specimens obtained after surgical treatment of hepatocellular carcinoma. J Gastroenterol Hepatol 2018;33:347-54.

11. Lee S, Kim SH, Lee JE, Sinn DH, Park CK. Preoperative gadoxetic acid-enhanced MRI for predicting microvascular invasion in patients with single hepatocellular carcinoma. J Hepatol 2017;67:526-34.

12. Wei H, Jiang H, Liu X, Qin Y, Zheng T, Liu S, Zhang $\mathrm{X}$, Song B. Can LI-RADS imaging features at gadoxetic acid-enhanced MRI predict aggressive features on pathology of single hepatocellular carcinoma? Eur J Radiol 2020;132:109312.

13. Chou CT, Chen RC, Lin WC, Ko CJ, Chen CB, Chen YL. Prediction of microvascular invasion of hepatocellular carcinoma: preoperative CT and histopathologic correlation. AJR Am J Roentgenol 2014;203:W253-9.

14. Tang Z, Yu D, Ni T, Zhao T, Jin Y, Dong E. Quantitative Analysis of Multiphase Contrast-Enhanced CT Images: A Pilot Study of Preoperative Prediction of Fat-Poor Angiomyolipoma and Renal Cell Carcinoma. AJR Am J Roentgenol 2020;214:370-82.

15. Dinapoli N, Barbaro B, Gatta R, Chiloiro G, Casà C, Masciocchi C, et al. Magnetic Resonance, Vendorindependent, Intensity Histogram Analysis Predicting Pathologic Complete Response After Radiochemotherapy 
of Rectal Cancer. Int J Radiat Oncol Biol Phys 2018;102:765-74.

16. Hu XX, Yang ZX, Liang HY, Ding Y, Grimm R, Fu CX, Liu H, Yan X, Ji Y, Zeng MS, Rao SX. Whole-tumor MRI histogram analyses of hepatocellular carcinoma: Correlations with Ki-67 labeling index. J Magn Reson Imaging 2017;46:383-92.

17. Ma X, Ouyang H, Wang S, Wang M, Zhou C, Zhao X. Histogram analysis of apparent diffusion coefficient predicts response to radiofrequency ablation in hepatocellular carcinoma. Chin J Cancer Res 2019;31:366-74.

18. Xu YS, Liu HF, Xi DL, Li JK, Liu Z, Yan RF, Lei JQ. Whole-lesion histogram analysis metrics of the apparent diffusion coefficient: a correlation study with histological grade of hepatocellular carcinoma. Abdom Radiol (NY) 2019;44:3089-98.

19. Huang YQ, Liang HY, Yang ZX, Ding Y, Zeng MS, Rao SX. Value of MR histogram analyses for prediction of microvascular invasion of hepatocellular carcinoma. Medicine (Baltimore) 2016;95:e4034.

20. Li H, Zhang J, Zheng Z, Guo Y, Chen M, Xie C, Zhang Z, Mei Y, Feng Y, Xu Y. Preoperative histogram analysis of intravoxel incoherent motion (IVIM) for predicting microvascular invasion in patients with single hepatocellular carcinoma. Eur J Radiol 2018;105:65-71.

21. Zhu F, Yang F, Li J, Chen W, Yang W. Incomplete tumor capsule on preoperative imaging reveals microvascular invasion in hepatocellular carcinoma: a systematic review and meta-analysis. Abdom Radiol (NY) 2019;44:3049-57.

22. Chernyak V, Fowler KJ, Kamaya A, Kielar AZ, Elsayes KM, Bashir MR, Kono Y, Do RK, Mitchell DG, Singal AG, Tang A, Sirlin CB. Liver Imaging Reporting and Data System (LI-RADS) Version 2018: Imaging of Hepatocellular Carcinoma in At-Risk Patients. Radiology 2018;289:816-30.

23. Xu M, Tang Q, Li M, Liu Y, Li F. An analysis of Ki-67 expression in stage 1 invasive ductal breast carcinoma using apparent diffusion coefficient histograms. Quant Imaging Med Surg 2021;11:1518-31.

24. Kim H, Park MS, Choi JY, Park YN, Kim MJ, Kim KS, Choi JS, Han KH, Kim E, Kim KW. Can microvessel invasion of hepatocellular carcinoma be predicted by preoperative MRI? Eur Radiol 2009;19:1744-51.

25. Reginelli A, Vacca G, Segreto T, Picascia R, Clemente A, Urraro F, Serra N, Vanzulli A, Cappabianca S. Can microvascular invasion in hepatocellular carcinoma be predicted by diagnostic imaging? A critical review. Future Oncol 2018;14:2985-94.

26. Pawlik TM, Delman KA, Vauthey JN, Nagorney DM, Ng IO, Ikai I, Yamaoka Y, Belghiti J, Lauwers GY, Poon RT, Abdalla EK. Tumor size predicts vascular invasion and histologic grade: Implications for selection of surgical treatment for hepatocellular carcinoma. Liver Transpl 2005;11:1086-92.

27. Omata M, Cheng AL, Kokudo N, Kudo M, Lee JM, Jia $\mathrm{J}$, et al. Asia-Pacific clinical practice guidelines on the management of hepatocellular carcinoma: a 2017 update. Hepatol Int 2017;11:317-70.

28. Zheng J, Chakraborty J, Chapman WC, Gerst S, Gonen M, Pak LM, Jarnagin WR, DeMatteo RP, Do RKG, Simpson AL; Research Staff in the Department of Surgery at Washington University School of Medicine. Preoperative Prediction of Microvascular Invasion in Hepatocellular Carcinoma Using Quantitative Image Analysis. J Am Coll Surg 2017;225:778-788.e1.

29. Dai H, Lu M, Huang B, Tang M, Pang T, Liao B, Cai H, Huang M, Zhou Y, Chen X, Ding H, Feng ST. Considerable effects of imaging sequences, feature extraction, feature selection, and classifiers on radiomics-based prediction of microvascular invasion in hepatocellular carcinoma using magnetic resonance imaging. Quant Imaging Med Surg 2021;11:1836-53.
Cite this article as: Wang X, Sun Y, Zhou X, Shen Z, Zhang H, Xing J, Zhou Y. Histogram peritumoral enhanced features on MRI arterial phase with extracellular contrast agent can improve prediction of microvascular invasion of hepatocellular carcinoma. Quant Imaging Med Surg 2022;12(2):1372-1384. doi: 10.21037/qims-21-499 